\title{
Correction to: Advances in Artificial Systems for Medicine and Education V
}

Zhengbing $\mathrm{Hu}$, Sergey Petoukhov, and Matthew He

\section{Correction to:}

Z. Hu et al. (Eds.): Advances in Artificial Systems for Medicine and Education $V$, LNDECT 120, https://doi.org/10.1007/978-3-030-92537-6

The original version of the book was published with incorrect volume number. This has been corrected in the updated version. 